Ann. Abeille, Ig67, 10 (4), 203-212.

\title{
L'EFFET DE GROUPE ET LA MORTALITÉ DES ABEILLES D’HIVER ET D'ÉTÉ, ISOLÉES ET GROUPÉES.
}

\author{
(i. SITBOA \\ Laboratoive de P'svchophysiologie, \\ Fiaculti des Sciences, 6r - Strasbourg
}

SOMMAIRE

Les abeilles isolées meurent beaucoup plus vite que celles groupées et ce, qu'il s'agisse d'abeilles d'hiver ou d'été. Le groupement joue donc un rôle important dans la mortalité, surtout pendant les premiers jours suivant l'éclosion, période pendant lacjuelle les échanges de nourriture sont très fréquents et, semble-t-il, indispensables

\section{IN'TRODUC'TION}

I)ans tme expérience antérieure ( $\lg 67$ a), une étude systématique de la mortalité des abeilles d'hiver isolées et groupées a montré que les premières mouraient plus vite que les secondes. Mais est-ce un phénomène spécial aux abeilles d'hiver? C'est ce que ce travail se propose de déterminer, tout en comparant l'évolution de la mortalité des 2 types d'abeilles, d'été et d'hiver.

\section{MATÉRIEI, E'T MÉTHODES} $1967 b)$.

Le type de cagettes utilisées pour l'isolement a longuement été décrit (PAIN, i966 ; Sitbon,

Une première série d'expériences a porté sur 80 cagettes ne contenant qu'une abeille et 8 en contenant 50 , et une deuxième série sur 80 cagettes à I abeille et 4 à $5^{\circ}$.

Toutes les abeilles proviennent de cadres de couvain, prélevés dans 5 ruches extérieures, et gardés en étuve à $32^{\circ} \mathrm{C}$. Les cadres ont été prélevés le 7 juin 1967 pour la ${ }_{1}$ ere série d'expériences 
et le 7 juillet 1967 pour la $2^{\mathrm{e}}$. Il s'agit donc d'abeilles naissantes, écloses en étuve et provenant de colonies différentes. Leur espérance de vie, dans les conditions naturelles, est, selon Maurizio (I96I $a$ ), de 25 à 35 jours en moyenne, avec un maximum de 60 à 70 jours.

Toutes les abeilles disposent d'eau pure et de candi à reine pendant toute la durée de l'expérience, et de pollen pendant les 7 premiers jours $\left({ }^{1}\right)$. Mais pour la première série, le pollen provenait d'une récolte de 1965 et de ce fait n'a exercé qu'un faible attrait. Au contraire les abeilles de la $2^{\mathrm{e}}$ expérience disposaient d'un mélange de pollen frais récolté entre le 23 et 28 juin 1967 sur 4 ruches $\left(^{2}\right)$. En outre, le pollen a été renouvelé tous les jours.

Dans les 2 expériences, les cagettes sont réparties sur les 8 rayons de 2 étuves métalliques déjà décrites $\left(\lg ^{67} b\right)$, à raison de 20 cagettes à I abeille et de 2 ou x à $5 \circ$, par rayon. La température de chaque étuve est de $32^{\circ} \mathrm{C} \pm \mathrm{I}^{\circ} \mathrm{C}$. Toutes les abeilles sont maintenues à l'obscurité.

Dans les groupes de 5 , les cadavres sont retirés au moment du contrôle de la mortalité, c'està-dire tous les jours à la même heure (II h).

\section{RÉSULTATS}

\section{Mortalité chez les abeilles d'été}

J'ai déjà montré que les différences de température existant entre les divers rayons d'une étuve sont sans importance; autrement dit, une variation de $\pm \mathrm{I}^{\circ} \mathrm{C}$ peut être négligée. C'est pourquoi dans les expériences présentes, je n'ai considéré que l'ensemble des individus d'une étuve en faisant abstraction du rayon sur lequel ils se trouvaient. C'est cette mortalité, exprimée en pourcentage, que traduisent les figures I et 2 .

Une fois de plus, ces figures montrent clairement une différence de mortalité importante entre abeilles isolées et groupées. En effet, pour les abeilles prélevées en juin (fig. I) les $50 \mathrm{p}$. Ioo ne sont atteints chez les groupées que le $3^{\mathrm{e}}$ jour, alors que la moitié des abeilles isolées a disparu le $I 5^{\mathrm{e}}$ jour. En outre, le $4 \mathrm{O}^{\mathrm{e}}$ jour, il ne reste plus que I,25 p. Ioo d'abeilles isolées contre 42,75 p. Ioo de groupées.

Ein ce qui concerne les abeilles prélevées en juillet (fig. 2), les différences de mortalité sont tout aussi marquées. La moitié des abeilles isolées a disparu le $2 \mathrm{I}^{\mathrm{e}}$ jour alors que chez les groupées les $50 \mathrm{p}$. Ioo ne sont atteints que le $40^{\mathrm{e}}$ jour.

Cette différence de mortalité passe par un maximum. Ainsi, pour les individus de juin, la différence de mortalité est la plus grande le $23^{\mathrm{e}}$ jour et atteint $60,75 \mathrm{p}$. Ioo. Pour les abeilles de juillet, le maximum est atteint le $34^{\mathrm{e}}$ jour avec 45,25 p. Ioo. Par ailleurs, les expériences ayant été poursuivies jusqu'à extinction de l'ensemble des abeilles, il est possible de calculer la durée de vie moyenne des abeilles isolées et groupées. Ces chiffres soulignent l'importance de la différence de mortalité. En effet, les abeilles isolées en juin et juillet vivent en moyenne respectivement I5,70 jours et 22,13 jours, alors que les abeilles groupées correspondantes tiennent, dans les mêmes conditions, 39,94 jours et 42,53 jours. La vie des abeilles groupées par 50, est donc en moyenne 2 fois plus longue que celle des isolées. Fn outre, les durées extrêmes de vie, notées en juin et juillet, sont respectivement de 48 et 67 jours pour les individus isolés et 83 et II 4 jours pour ceux groupés.

Notons au passage que, comme chez les abeilles d'hiver, la mortalité des individus isolés semble se stabiliser en fin d'expérience. J'ai déjà attribué ce phénomène à un

(1) Le pollen n'a été dispensé que pendant 7 jours puisqu'il est admis que les jeuncs abeilles n'en consomment que les 4 à 5 premiers jours de leur vie.

(2) Je remercie la station de Recherches sur l'Abeille et les Insectes sociaux de Bures-Sur-Yvette (Lssonne) qui a bien voulu me fournir tout le pollen indispensable à l'expérience. 


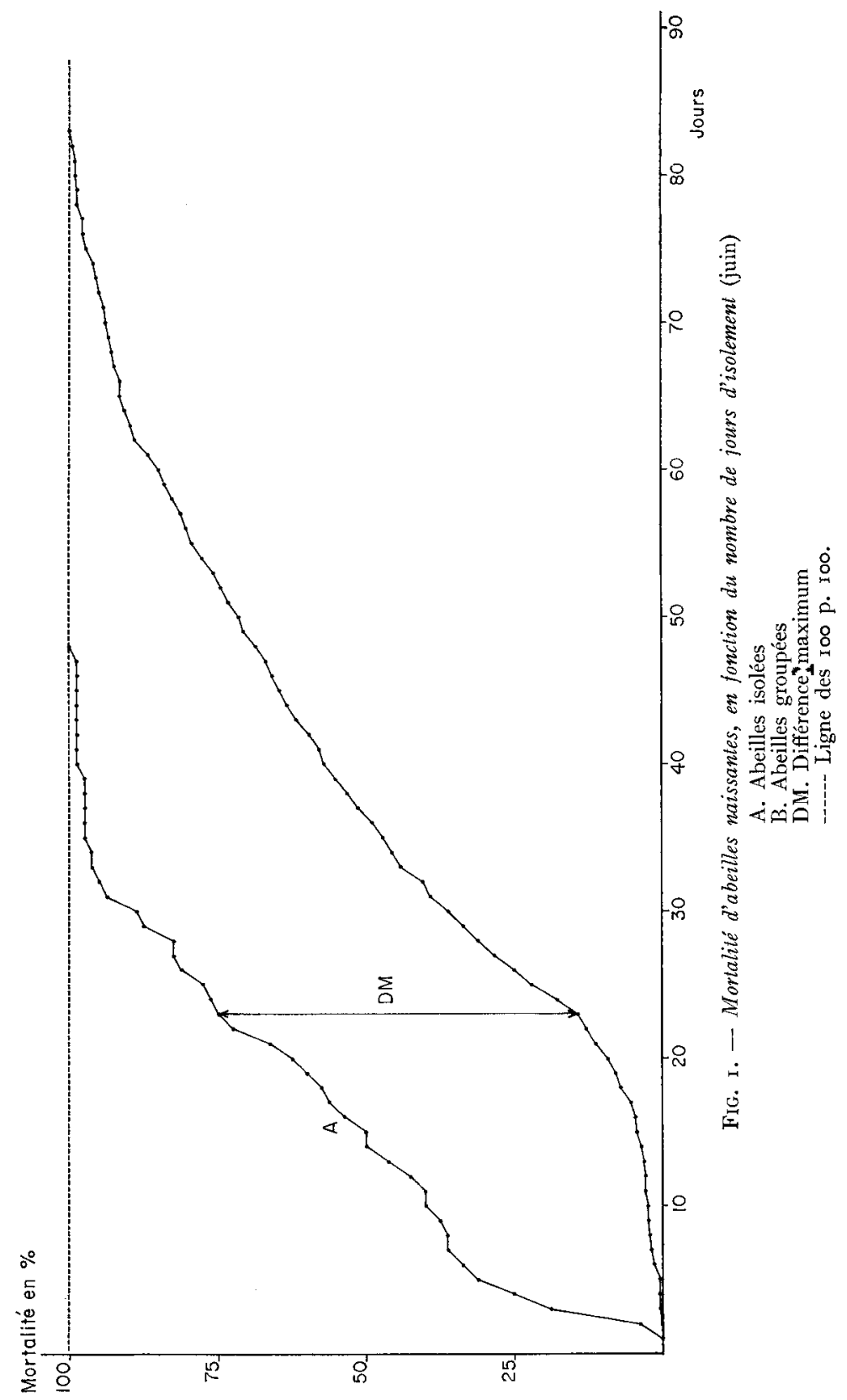




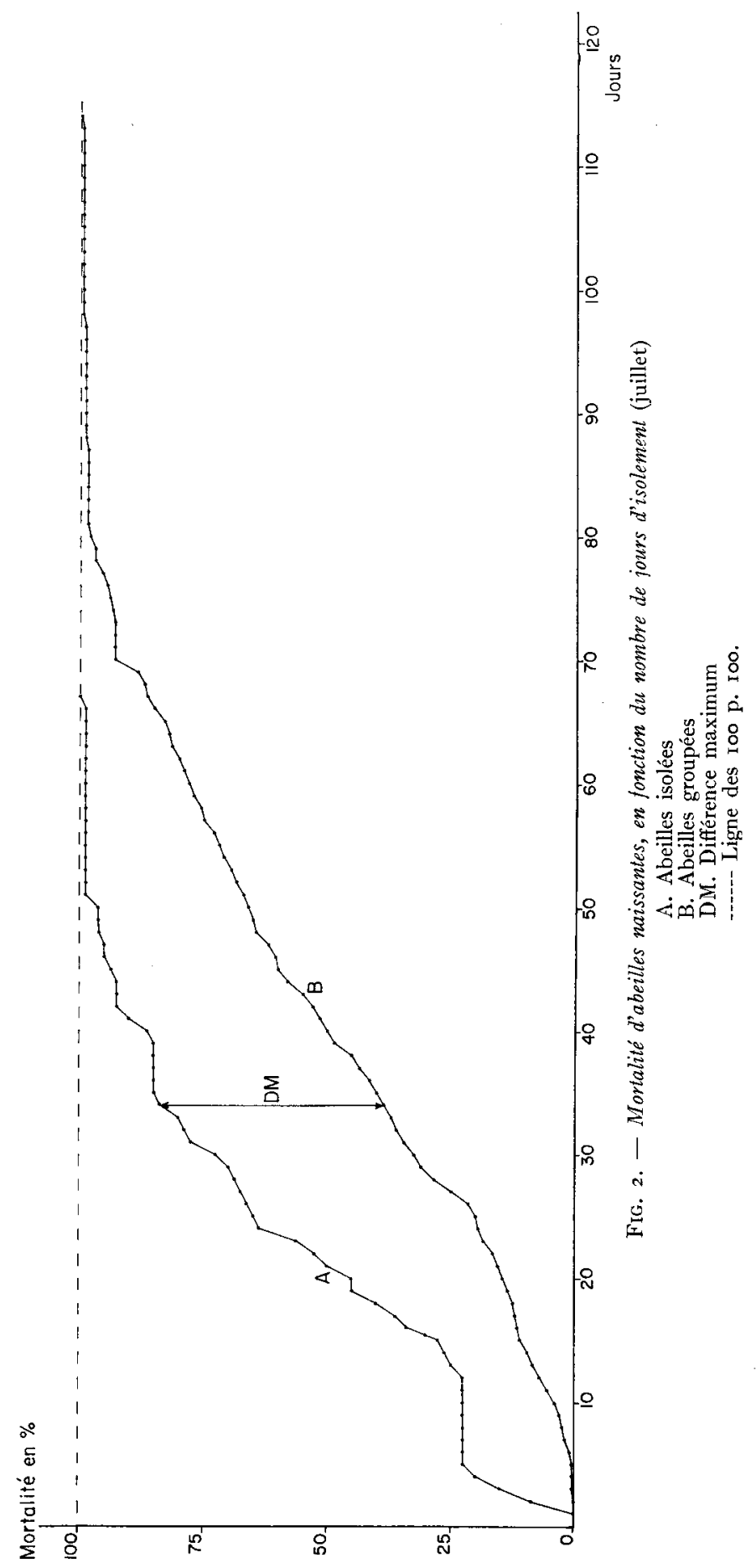


artefact, le nombre d'abeilles décédées étant proche des roo p. roo. Mais, comme on peut le constater, cette stabilisation couvre une période de Io à I 5 jours. Cela laisse supposer qu'une catégorie d'abeilles, numériquement très faiblement représentée, supporte mieux l'isolement. Il est évident qu'il s'agit là d'une hypothèse et qu'une étude plus approfondie s'impose.

Par ailleurs, il ressort des courbes obtenues que les abeilles isolées en juin ont une survie plus courte que celles de juillet. Mais je pense que cette différence est essentiellement due aux conditions expérimentales. En effet, comme je l'ai déjà dit, les abeilles de juin ont đisposé de pollen récolté longtemps avant l'expérience et de ce fait très faiblement consommé, alors que les abeilles de juillet ont eu du pollen frais et fréquemment renouvelé dont la consommation est nettement plus importante. Or, de très nombreux auteurs dont DE Groo'T (I953), Maurizio (I946-I954) ont montré que la consommation de pollen améliore très sensiblement la longévité des ouvrières sans reine. Cette expérience garde cependant toute sa valeur puisqu'il s'agit de comparer des abeilles groupées à des abeilles isolées, placées dans les mêmes conditions, en l'occurrence disposant du même pollen.

\section{Comparaison de la mortalité d'abeilles d'hiver et d'été}

I'expérience portant sur les abeilles d'hiver ( $\left.\operatorname{Ig67}_{7} b\right)$ n'ayant été poursuivie que pendant $2 \mathrm{r}$ jours, la comparaison ne sera donc possible que pour cette période expérimentale. C'est donc l'évolution de la mortalité des abeilles d'hiver et d'été en fonction du groupement, de cette période, que représente la figure 3 .

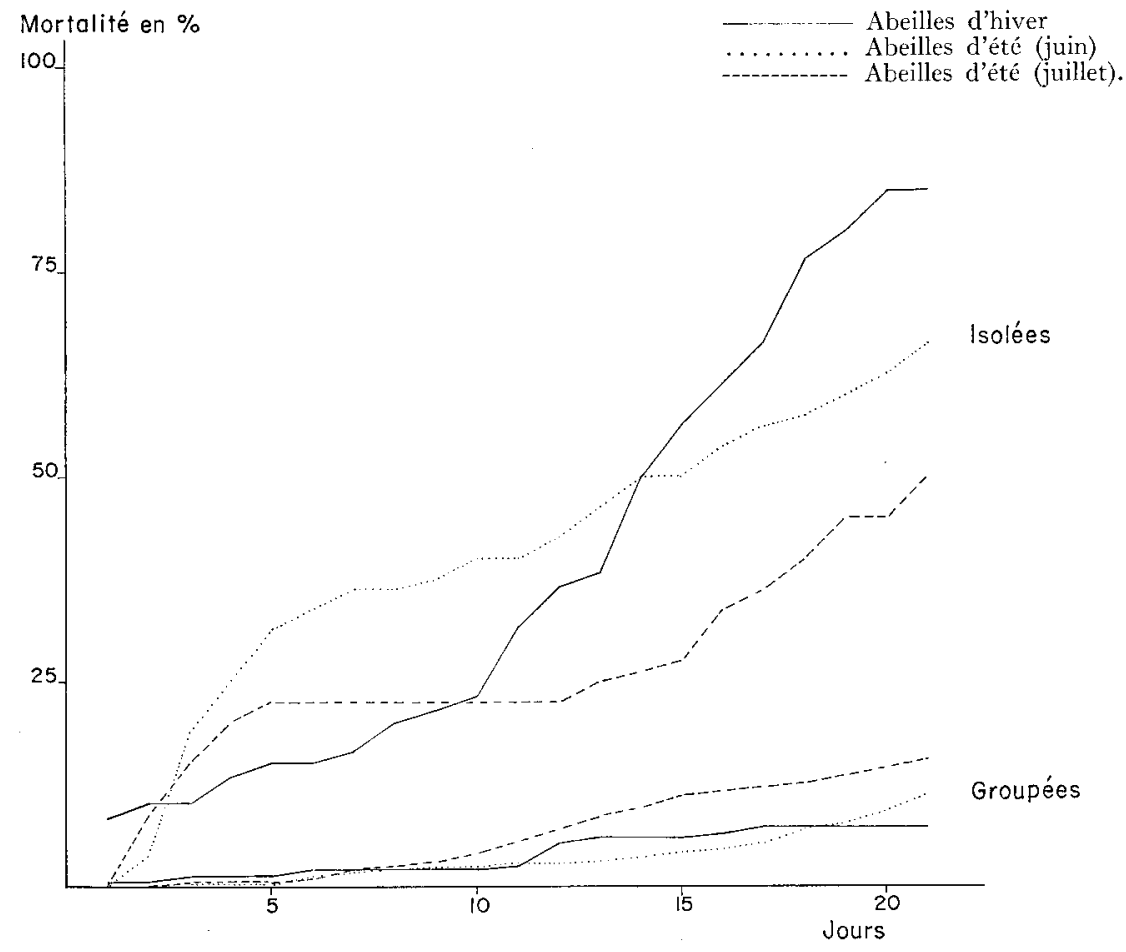

FIG. 3. - Mortalité en fonction du nombre de jours d'isolement chez les abeilles isolées et groupées. 
De ces courbes, il ressort les points suivants :

a) le $\mathrm{I}^{\mathrm{er}}$ jour d'isolement, la mortalité des abeilles d'été, isolées ou groupées, est nulle alors que chez les abeilles d'hiver elle est de 8,3 p. Ioo et 0,6 p. Ioo, respectivement. Ceci peut s'expliquer si l'on tient compte que, dans le $2^{\mathrm{e}}$ cas, les abeilles traitées sont âgées de 3 mois environ et habituées à vivre en groupe. I) ce fait, toute manipulation engendre des perturbations et une grande agitation pouvant aller jusqu'à la mort. Cette agression expérimentale est pratiquement absente pour les abeilles des expériences d'été. Ein effet, il s'agit d'abeilles naissantes, très peu actives et ne réagissant que très faiblement aux diverses manipulations subies. Ceci explique d'ailleurs qu'il n'y ait pas de différence de mortalité entre les abeilles d'été, isolées et groupées, le I $^{\text {er jour ; }}$

b) chez les abeilles d'été isolées, la mortalité croit rapidement entre le $2^{\mathrm{e}}$ et le

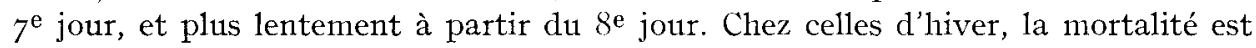
nettement inférieure pour la première période et croît régulièrement pendant toute la durée de l'expérience. Autrement dit, pour les abeilles d'hiver, la courbe de mortalité est sensiblement une parabole dont la première partie, comme je l'ai déjà expliqué ( 1967 b) traduit la juxtaposition de 2 phénomènes : agression expérimentale et isolement. Au contraire, pour les abeilles d'été, les courbes ne peuvent être assimilées à des droites qu'à partir du Io $^{\mathrm{e}}$ jour. C'est ce que traduisent les courbes simplifiées de la figure 4 .

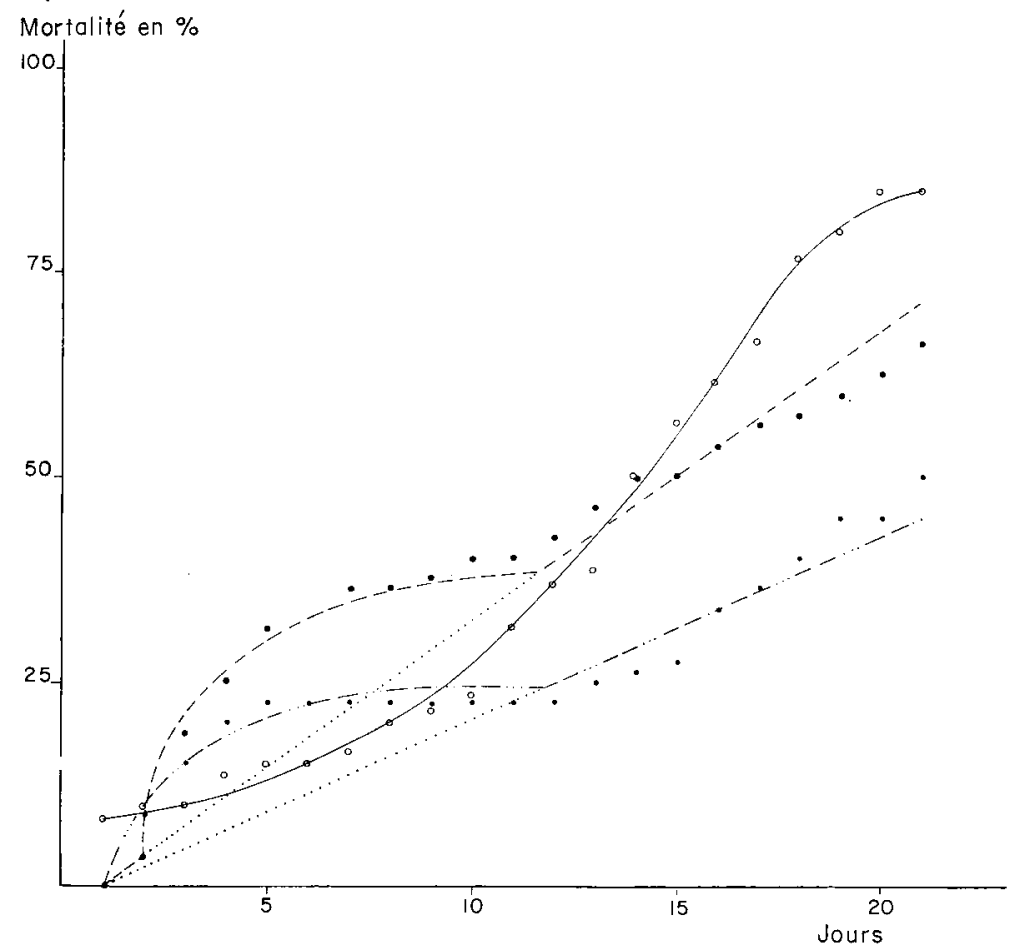

FIG. 4. - Courbes simplifiées de la mortalité, en fonction du nombre de jours d'isolement, chez des abeilles isolées

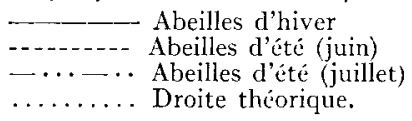


Pour les abeilles groupées, la mortalité évolue régulièrement dans les 3 cas étudiés. En particulier, pour les abeilles d'été, la mortalité est pratiquement nulle pour la période du $2^{\mathrm{e}}$ au $7^{\mathrm{e}}$ jour.

En résumé, pendant la période du $2 \mathrm{e}$ au $\mathrm{Fe}^{\mathrm{e}}$ jour, la mortalité des abeilles d'été isolées suit des lois spéciales.

Or, PERShad (I966) a montré, à l'aide de nourriture marquée au phosphore 32, chez des ouvrières de même âge, que les échanges de nourriture sont nuls à l'âge d'un jour, passent par un maximum vers l'âge de 4 jours, pour redevenir très faibles à 1'âge de 7 jours. Ces constatations s'appliquent donc à mon matériel d'été, mes 2 expériences ayant porté sur des abeilles naissantes. En effet, le I er jour, les échanges de nourriture étant nuls et l'agression expérimentale absente, la mortalité des abeilles d'été, groupées ou isolées, est nulle. Au contraire, la mortalité des abeilles isolées est particulièrement rapide $\mathrm{du} 2^{\mathrm{e}}$ au $7^{\mathrm{e}}$ jour, avec un début de stabilisation vers le $6^{\mathrm{e}}$ jour, ce qui correspond à la période essentielle des échanges. Autrement dit, ces échanges de nourriture étant impossibles, il s'ensuit une forte mortalité avec un maximum vers le $4^{\mathrm{e}}$ ou $5^{\mathrm{e}}$ jour. Ceci explique pourquoi la mortalité, pendant la dite période, est pratiquement nulle chez les abeilles groupées et nettement inférieure chez les abeilles d'hiver isolées, âgées de 3 mois. Cette période passée, la mortalité devient plus régulière et la courbe sensiblement rectiligne.

Il semble donc que les abeilles d'été naissantes aient besoin de «nourriture trophallactique "pendant les 5 à 6 premiers jours de leur vie, sous peine de succomber;

c) chez les abeilles groupées, la mortalité est légèrement plus forte pour les abeilles d'été. Au contraire, chez les abeilles isolées, ce sont les abeilles d'hiver qui meurent plus vite.

En conclusion nous retiendrons que les manipulations expérimentales, ressenties si vivement par les abeilles d'hiver, perturbent peu les abeilles naissantes et qu'il existe entre le $2^{\mathrm{e}}$ jour et le $7^{\mathrm{e}}$ jour un rapport étroit entre la mortalité des abeilles naissantes, isolées en été, et la trophallaxie.

\section{DISCUSSION}

\section{La mortalité des abeilles groupées}

Les auteurs se sont beaucoup plus occupés de la mortalité des abeilles groupées qu'isolées, en fonction de l'influence de différents facteurs. Les travaux dans ce domaine sont très nombreux. Pour ma part, j'ai tenu compte des résultats antérieurs en sélectionnant les facteurs susceptibles d'améliorer la longévité. Ainsi, j'ai travaillé sur des groupes de $5^{\circ}$ abeilles car CHauvin (I952) a montré que c'est à partir de 30 individus que 1'on pouvait faire des études convenables sur la durée de vie des abeilles encagées. La température choisie est de $32^{\circ} \mathrm{C}$, car il s'est avéré à la suite des travaux de Melampy et McGregor (I939) et Maurizio (I946) que la durée de vie moyenne des abeilles d'été et d'hiver est plus longue entre 30 et $35^{\circ} \mathrm{C}$. Les abeilles ont disposé d'eau pure et fréquemment renouvelée tout au long de l'expérience. En effet, WOODRow (I935) a montré que les abeilles nourries de candi vivent plus longtemps lorsqu'elles reçoivent de l'eau et MaUrizio que des abeilles assoiffées 
vivent trois fois moins que les autres. Toujours selon MaURIzIo, le candi améliore la longévité des abeilles d'été, mais pour les abeilles d'hiver le sirop de sucre est préférable. Cependant, j'ai nourri toutes les abeilles an candi, et ceci pout 2 raisons : d'une part, il fallait adopter des conditions expérimentales identiques afin de pouvoir comparer les résultats, et d'autre part, les abeilles étant claustrées, elles ne défèquent pas. Un aliment solide est plus compatible avec ce fait qu'un aliment liquide (CHAUVIN, I952).

En outre, pour réaliser expérimentalement, aussi parfaitement que possible, les conditions physiques de la ruche, les abeilles disposent d'une plaquette de cire gaufrée.

Dans ces conditions expérimentales, les résultats obtenus sont très satisfaisants. En effet, d'une part le comportement des abeilles paraît tout à fait normal puisqu'elles étirent des alvéoles et d'autre part la durée de vie moyenne des abeilles d'été, étudiées en juin et juillet, est respectivement de 40 et 43 jours. Par ailleurs, la moitié des 2 populations a disparu en 36 et 40 jours. Or, Maurizio (I946) n'a obtenu en été qu'une longévité moyenne de 25,2 jours et une disparition des $50 \mathrm{p}$. Ioo des abeilles récoltées de mai à juin après 20 à 25 jours seulement (pour celles récoltées de septembre à marsavril, il faut 36 jours). Chauvin (I952) note aussi une disparition de la moitié de la population en 20 à 25 jours alors que ses abeilles ont été récoltées en mars. Mais l'auteur pense que cette différence avec les résultats de MAURIzIO peut s'expliquer par une saison apicole plus avancée à Bures qu'à Liebefeld.

En résumé, les conditions expérimentales choisies m'ont donc permis d'améliorer la durée de vie moyenne des abeilles d'été d'une quinzaine de jours et même de dépasser leur espérance de vie dans les conditions naturelles, espérance de vie qui est de 25 à 35 jours pour des abeilles de mai à août (MAurızIo, I96I). Ce faitn'est d'ailleurs pas surprenant. Ėn effet, Maurizio (I96r) a montré que, dans les conditions naturelles, des abeilles d'été peuvent vivre plusieurs mois quand elles n'ont pas de couvain à entretenir. Mes expériences montrent que ceci est aussi valable pour des abeilles claustrées.

Par ailleurs, cette expérience a révélé une différence de mortalité entre abeilles groupées d'hiver et d'été. Ceci confirme les travaux de MaUrizio (I946) qui obtient des durées de vie moyenne de 23 à 25 jours en été et de 35 à 37 jours en hiver, et de BERTHOLF (I942) qui a montré que la longévité des abeilles d'hiver est légèrement supérieure à celle des abeilles d'été. Mais notons que ce dernier a travaillé sur des abeilles gardiennes d'âge inconnu. Je regrette fortement de ne pas avoir poursuivi plus longtemps mon étude sur les abeilles d'hiver, car il aurait été intéressant de savoir si statistiquement cette différence de mortalité que je qualifie de légère est significative ou non.

\section{La mortalité des abeilles isolées}

Les travaux sur la mortalité des abeilles isolées sont moins nombreux et les conclusions contradictoires.

En effet, Grassé et Chauvin (I944) et Chauvin (1952) notent des survies moyennes de l'ordre de 5 à 6 jours, tout comme WeIss (I930) et K. V. FRIsch (I953) et concluent que les abeilles isolées meurent plus vite que celles groupées. De même Mlle PaIN (I 960) signale que le nombre d'abeilles vivant dans les cagettes d'isolement agit sur la durée de vie des insectes et obtient une durée moyenne de vie de 7 jours. Cependant, elle obtient aussi, exceptionnellement, des survies de I9 jours. 
Au contraire, HeUSNER (Ig64) note que des butineuses isolées peuvent vivre jusqu'à 3 semaines et conclut que les survies observées sont comparables à l'espérance de vie normale, ce qui sous-entend que le groupement est sans effet sur la mortalité.

Pour ma part, dans une expérience antérieure (I967), j'ai obtenu des survies supérieures à celles observées par CHAuvin et GRAssé et j'en ai d'ailleurs donné l'explication. Mais j'ai également montré que l'effet du groupe sur la mortalité est indiscutable. Ce travail ayant porté sur đes abeilles d'hiver, je l'ai recommencé en été pour me placer dans des conditions expérimentales aussi proches que possible de celles de HEUSNER. Ces nouvelles expériences confirment que la durée moyenne de vie est supérieure à celle obtenue par ChAUvin et Grassé. Mais contrairement à ce que pense HeUSNER les abeilles isolées ici encore meurent beaucoup plus vite que celles groupées. Donc la survie évoquée par HéusNER est acceptable, encore qu'il aurait été intéressant de préciser sur quels pourcentages ces observations ont été faites, mais n'exclut nullement l'existence d'un effet du groupement sur la survie. D'ailleurs pour en discuter valablement, il eût fallu comparer la mortalité des abeilles isolées et groupées dans les mêmes conditions et non, comme il le fait, la mortalité des abeilles isolées dans des conditions artificielles à celle d'abeilles vivant en groupe dans des conditions normales. En effet, mes expériences montrent qu'en étuve la survie moyenne des groupes est supérieure à l'espérance de vie d'abeilles vivant dans des conditions naturelles et devant entretenir du couvain (MAURIZIO).

Il est donc exact qu'une abeille isolée "peut vivre jusqu'à 3 semaines " et même bien plus, mais il est aussi irréfutable que le groupement joue un rôle très important dans la mortalité.

Par ailleurs, mes expériences montrent que les abeilles d'été supportent mieux l'isolement que les abeilles d'hiver. Ceci confirme les travaux de Chauvin (I952) qui signale que la survie moyenne des abeilles isolées est de 6 jours sans grande variation de part et d'autre de ce chiffre au cours de l'année, mais note cependant qu'en janvier et février la durée moyenne de vie est nettement plus courte.

Par contre, aucun auteur, à ma connaissance, ne fait allusion à la nécessité de " nourriture trophallactique " pendant les premiers jours de la vie de jeunes abeilles. A ce propos, il convient de rappeler que les premiers jours d'expériences, la mortalité des abeilles isolées en été est plus forte que celle des abeilles d'hiver. Cépendant, je ne pense pas que cette différence soit à imputer au fait qu'il s'agit d'abeilles d'hiver et d'été, mais plutôt d'abeilles âgées et naissantes. Autrement dit un phénomène analogue devrait être décelable sur des abeilles d'hiver naissantes.

\section{CONCLUSION}

Ces expériences me permettent de généraliser les résultats trouvés dans les expériences précédentes ( 1967 ). Les abeilles isolées meurent beaucoup plus vite que celles groupées et ce, qu'il s'agisse d'abeilles d'hiver ou d'abeilles d'été. Eilles me permettent en outre de mettre en évidence que le groupement joue un rôle important dans la mortalité, surtout pendant les premiers jours suivant l'éclosion, période pendant laquelle les échanges de nourriture sont très fréquents et, semble-t-il, indispensables. 


\section{SUMMARY}

\section{GROUPING AND MORTALITY OF WTNTER AND SUMMER BEES, IS OLATED OR GROUPED}

In both winter and summer, isolated bees die much more quickly than those in groups. Grouping thus plays an important part in mortality. This is especially true during the first days after hatching, a period when food exchanges are very frequent and, it seems indispensable.

\section{ZUSAMMENFASSUNG}

\section{GRUPPENEFFEKT UND MORTALITÄT VON EINZELNEN UND GRUPPIERTEN WINTER-UND SOMMERBIENEN}

Die isolierten Bienen sterben viel schneller als die gruppierten, gleich, ob es sich um Winter. oder Sommerbienen handelt. Die Gruppierung spielt also bei der Sterblichkeit eine bedeutende Rolle, besonders in den ersten Tagen nach dem Auschlüpfen, eine Zeit, in der der Nahrungsaustausch sehr häufig und anscheinend unerlässlich ist.

\section{RÉFÉRENCES BIBLIOGRAPHIQUES}

Bertholf L. M., 1942. Eiffect of certain biological factors on the longevity of caged bees. J. econ. Enlomol., $35(6), 887-891$.

Chauvin R., 1952. Sur le déterminisme de l'effet de groupe chez les abeilles. Physiol. comp. Oecol., 1, i-7. DE Groot A. P., I953. Protein and aminoacid requirements of the honeybee (A. M. L.). Physiol. comp. Oecol., 3, I $97-285$.

Frisch K. v., 1953. Aus dem Leben der Bienen. I vol. 159 p., Springer, Berlin.

Grassé P. P., Chauvin R., i944. L'effet de groupe et la survie des neutres dans les sociétés animales. Rev. sci., $\mathrm{Y}, 46 \mathrm{r}-464$.

Heusner A, Stussi Th., rg64. Métabolisme énergétique de l'abeille isolée : son rôle dans la thermorégulation de la ruche. Insectes soc., 11 (3), 239-266.

Maurizio A., i946. Beobachtungen über die Lebensdauer und den Futterverbrauch gefangen gehaltener Bienen. Beih. Schweiz. Bienenztg., 2 (13), I-44.

Matzrzio A., I 954. Pollenernährung und Lebensvorgänge bei der Honigbiene (Apis mellifica L.). Landw, Jb. Schroeiz., $68(2)$, II $5^{-182}$.

Maurizio A., i96i a. Lebensdauer und Altern bei der Honigbiene (Apis mellifica L.). Geronlologia, 5, IIO-I 28.

Melampy R. M., Mc Gregor S. E., 1939. Nutritional value of certain foods for the adult honeybee. J. econ. Entomol., 32, $721-725$.

PAIN J,, i960. De l'influence du nombre des abeilles encagées sur la formation des œufs dans les ovaires de l'ouvrière. C. R. Acad. Sci., 250 (I 4), 2629-2631.

Pain J., i966. Nouveau modèle de cagettes expérimentales pour le maintien d'abeilles en captivité. Ann. Abeille, 9 (I), 7 r-76.

PERshad S. B., ig66. L'influence de l'âge sur les échanges de nourriture entre les ouvrières d'abeilles $A p i s$ mellifica. Insectes soc., 13 (4), $3^{2} 3^{-328 .}$

Sitbon G., rg67 a. Action de l'effet de groupe sur la mortalité et l'alimentation des abeilles en hiver. $C . R$. Acad. Sci., 264, 2035-2038.

Sitbon G., i $967 b$. L'effet de groupe chez l'Abeille. I. L'Abeille d'hiver, survie et consommation de candi des abeilles isolées ou groupées. Ann. Abeille ro, (2) $67-82$.

WeISS G., 1930. Sur certaines conditions de mort et de survie chez les abeilles et chez d'autres insectes en captivité. C. R. Soc. Biol., Paris, 105, 571-573.

Woodrow A. W., I935. Some effects of relative humidity on the length of life and food consumption of honeybees. J.econ. Entomol, 28, 565-568. 